\title{
Do imitation problems reflect a core characteristic in autism? Evidence from a literature review
}

\author{
Marleen Vanvuchelen a,b,c, ${ }^{*}$, Herbert Roeyers d, Willy De Weerdt b \\ a Department Health Care, PHL University College, Belgium \\ ๖ Department of Rehabilitation Sciences, K.U. Leuven, Belgium \\ cDepartment of Rehabilitation Sciences, V.U.B., Belgium \\ d Research Group Developmental Disorders, Ghent University, Belgium
}

\begin{abstract}
A B S T R A C T
Although imitation problems have been associated with autism for many years, the issue if these problems are a core deficit in autism remains subject of debate.

In this review article, the question if autism imitation problems fulfil the criteria of uniqueness, specificity, universality, persistency, precedence and broadness is explored and discussed. Findings of this review suggest that there is only partial evidence for the idea that imitation problems are unique, specific and broad to autism, and that these problems are long-lasting and persistent. In addition, imitation problems seem not to be universal in autism at an early age. Mental and motor impairment may affect imitation performance but they do not seem to explain imitation problems in a sufficient way.
\end{abstract} Recommendations for clinical practice are formulated. 


\section{Introduction}

Autistic disorder and related autism spectrum disorders (hereafter "autism") are neurodevelopmental disorders characterised by impairments in social interaction, in communication skills and in behaviour, which is restricted and repetitive. The onset of the impairment is before 3 years of age (American Psychiatric Association, 2000). One autism associated feature requiring more elucidation is problems in motor imitation (hereafter "imitation"). The DSM-IV-TR includes imitation problems among the diagnostic criteria of autism, but restricts these to a lack of social imitative play (American Psychiatric Association, 2000). A clear picture of imitative abilities in individuals with autism is confounded by the heterogeneity of the clinical phenotype (Levy, Mandell, \& Schultz, 2009), the lack of a consistent and operational definition of imitation and the wide variability across the types of imitation tasks (Sevlever \& Gillis, 2010). The term imitation has many definitions. There are however two connotations in which it is most commonly used. The first defines imitation as the capacity of an individual to replicate an observed motor act. The observer's perception of the demonstrator's behaviour causes similar behaviour in the observer (Prinz, 2002). The more unambiguous definition of imitation requires that a novel action be learned by observing another perform it. In addition to novelty, the imitation requires a means/ends structure. The observer copies the actors' means of achieving his result (Tomasello, Carpenter, Call, Behne, \& Moll, 2005).

Imitation problems have been associated with autism for many years. Rogers and Pennington (1991) were the first to review these problems, examining seven studies. Their influential paper found strong evidence for the existence of a deficit affecting imitation of simple body movements, as well as imitation of actions with symbolic meaning. The authors suggested that imitation is one of the core neuropsychological deficits in autism, present very early in development, with possible cascading effects on social relations, interactions, and learning throughout the life span (Rogers \& Pennington, 1991). In the same period, Smith and Bryson (1994) carried out a review of 15 studies conducted up to that point. They concluded that children with autism might show different patterns of imitative deficit according to their functional level and that the imitation deficit, rather than being primary, might be secondary to more basic, non-social processing deficits (Smith \& Bryson, 1994). In 1999, Rogers carried out a further review of the most rigorously designed published experimental studies of imitation in autism and found again strong evidence supporting the existence of a primary imitation deficit in autism (Rogers, 1999). In a comprehensive review Williams and colleagues (2004) identified 124 articles concerning autism and imitation up to March 2002. They reviewed in depth 21 experimental studies. When studies where chronological age and IQ were not controlled for were excluded, 12 studies remained. Only one of these studies did not report imitation problems in the autistic group when compared to controls. Williams and colleagues pooled the findings from these studies, involving 196 individuals with autism, using the Logit method. They calculated the combined $p$-value of group differences with respect to imitation problems to an appropriate control group, resulting in a $p$ value of .00002 (Williams, Whiten, \& Singh, 2004).

To understand the nature of the imitation problems in autism, it is important to address the question if these problems are a core deficit in autism. A deficit is considered to be a core characteristic if it is unique to autism, specific and universal (Sigman, Dijamco, Gratier, \& Rozga, 2004). In addition, a deficit may fulfil the criteria of persistency (Hobson \& Lee, 1999), precedence (Rogers, 1999) and broadness. What follows is an incisive review of imitation studies in autism. This review is restricted to experimental studies of elicited imitative behaviour because in observational studies of spontaneous imitative behaviour ([Bird et al., 2007], [Knott et al., 2007] and Warreyn et al., 2005 P. Warreyn, H. Roeyers and I. De Groote, Early social communicative behaviours of preschoolers with autism spectrum disorder during interaction with their mothers, Autism 9 (2005), pp. 342-361.

Full Text via CrossRef | View Record in Scopus | Cited By in Scopus (8)[Warreyn et al., 2005]) or in parent reports (Thurm, Lord, Lee, \& Newschaffer, 2007) conditions for eliciting imitation may differ with respect to 
motivation and attention. However, research findings consistently suggest that persons with autism imitate significantly better in a structured-elicited than in a naturalistic-spontaneous imitation condition ([Ingersoll, 2008], [McDuffie et al., 2007], [McIntosh et al., 2006] and [Whiten and Brown, 1999]).

\section{Are imitation problems unique to autism?}

The requirement that the deficit is unique leads to studies that compare the functioning of groups with autism to control groups with different disabilities and matched for age and level of development (Sigman et al., 2004). Findings from most case-control studies would appear to support the contention that imitation problems are unique to autism (see reviews of [Rogers and Pennington, 1991], [Rogers, 1999], [Smith and Bryson, 1994] and [Williams et al., 2004]). The non-autistic comparison groups in these case-control studies consisted of persons with minimal brain damage MBD (DeMeyer et al., 1972), language disorders ([Bartak et al., 1975], [Jones and Prior, 1985], [Smith and Bryson, 1998] and [Stone et al., 1997]), hearing impairment (Stone, Lemanek, Fishel, Fernandez, \& Altemeier, 1990), mental disability ([Hammes and Langdell, 1981], [Jones and Prior, 1985], [Perra et al., 2008], [Rogers et al., 2010], [Roeyers et al., 1998], [Stone et al., 1990], [Vanvuchelen et al., 2007a] and [Vanvuchelen et al., 2007b]), developmental coordination disorders (DCD; [Dewey et al., 2007] and [Green et al., 2002]), ADHD ([Dewey et al., 2007] and [Ohta, 1987]), DCD plus ADHD (Dewey et al., 2007) as well as healthy persons ([Perra et al., 2008], [Rogers et al., 2010], [Stone et al., 1990], [Stone et al., 1997], [Vanvuchelen et al., 2007a] and [Vanvuchelen et al., 2007b]).

The research group of Perra (2008) used an alternative method to investigate whether imitative tasks can be used to discriminate children with autism from chronological age-matched typically developing and older mentally delayed peers. They used discriminant function analyses to the performance of these groups on three imitation tasks and on tests of fine motor performance, motor planning, verbal ability and theory of mind. When differences in fine motor performance and verbal ability were accounted for, differences in the imitation tasks and the Theory of Mind test discriminated the autism group from the other groups. Further analyses revealed that inclusion of imitative tasks increased the specificity and sensitivity of autism classification and that imitative tasks considered alone were able to reliably discriminate the three groups (Perra et al., 2008). However, the quality of a test to distinguish severely affected children, as children with autism are, from healthy children and children with a general mental delay, tells us nothing about the clinical utility of the test. Moreover, case-control study designs which compare the target condition with people who have clearly other disorders, overestimate the diagnostic utility of a test (Jaeschke, Guyatt, \& Sackett, 1994). The diagnostic utility of an imitation test to determine whether or not a patient has autism should be investigated in a random sample of the indicated population, i.e., patients suspected of autism. Vanvuchelen et al. (2010a) studied imitation aptitude in a referral sample of children suspected of autism with the use of a standardised imitation test. They found that preschoolers with non-spectrum developmental disorders who were previously suspected of autism were less retarded in procedural imitation, i.e., imitation of actions with objects, than preschoolers with confirmed autism, but the difference was only marginally significant ( $p$-value of 0.054 ). The authors concluded that only a procedural imitation delay, and not a bodily imitation delay, that goes beyond the nonverbal mental retardation may be a significant predictor of autism (Vanvuchelen et al., 2010a). Overall, there was some, but no clear evidence that imitation problems are unique to autism.

\section{Are imitation problems specific in autism?}

To establish the specificity of the deficit, research is carried out that contrasts different functions and abilities across groups, testing the hypothesis that some functions are deficient in the autism group while others are not 
involved, or that imitation problems are specific rather than being part of a general problem (Sigman et al., 2004). Results of Williams and colleagues' (2004) meta-analysis revealed that mental delay account for some impairment but by no means all of it (Williams et al., 2004).

Another question related to the specificity criterion is if persons with autism have imitation problems regardless of their general motor problems? DeMeyer and colleagues (1972) suggested that the inability of a person with autism to imitate reflects a disturbance of the capacity to formulate and execute an intentional motor plan in spite of intact motor abilities. Jones and Prior (1985) suggested that persons with autism do not have much difficulty with imitation, but with generalised motor planning and execution. More recently, the research group of Mostofsky (2006) suggested that autism is associated with a generalised praxis deficit, rather than with a deficit specific to imitation (Mostofsky et al., 2006). General motor problems are a well-recognised feature of autism. In a comprehensive review Fournier and colleagues (2010) identified 83 autism studies focused on motor coordination, arm movements, gait, or postural stability deficits. Data extraction involved between-group comparisons for autism and typically developing controls. Rigorous meta-analysis techniques determined a significant standardised mean difference effect equal to $1.20(p<0.0001)$. This large effect indicated substantial motor coordination deficits in the autism groups across a wide range of behaviours. The authors concluded that motor coordination deficits are an underlying cardinal feature of autism (Fournier et al., 2010).

Several studies have examined the relationship between motor and imitation performances with the use of formal motor tests ([Green et al., 2002], [Perra et al., 2008], [Rogers et al., 2003], [Smith and Bryson, 1998], [Vanvuchelen et al., 2007a], [Vanvuchelen et al., 2007b] and [Zachor et al., 2010]). These studies demonstrated that the diagnosis of autism continued to be related to imitation, even after the variance associated with motor performance was removed. However, problems with formal motor tests are the imitative demands inherent in their instructions. Dzuik and colleagues (2007) investigated the association between dyspraxia and basic motor deficits in children with autism without mental impairment compared to typically developing peers without using of formal motor tests. Dyspraxia was defined as an impairment of skilled gestures, including gestures to imitation. Basic motor examination consisted of the evaluation of subtle neurological signs, which not rely on imitation abilities. Children with autism had significantly more praxis problems and subtle neurological signs than typically developing peers. The total number of errors from the praxis examination was a significant predictor of total ADOS-G scores, even after controlling for basic motor abilities. For this reason, the authors concluded that dyspraxia in the autism group cannot entirely be accounted for by basic motor deficits. They speculated that basic motor impairments and impaired praxis performance, including imitation problems, may be epiphenomena in autism, i.e., that a common neurological abnormality contributes to both basic motor deficits and to dyspraxia observed in autism (Dziuk et al., 2007). In contrast, in Zachor et al.'s study (2010) imitation abilities of children with autism correlated significantly only with autism severity measures. Motor abilities did not correlate with imitation abilities nor with autism severity (Zachor et al., 2010). To circumvent the possible influence of mental problems in children with autism, Vanvuchelen and colleagues (in pressa) used nonverbal mental age as a reference criterion. Imitation and motor delay were defined as an imitation and motor development below the expectation based on the child's nonverbal mental age. The authors used a standardised imitation scale, the Preschool Imitation and Praxis Scale (PIPS) (Vanvuchelen et al., 2010b). The imitation age-equivalent scores of the PIPS offered the possibility to determine imitation delay in relation to the children's nonverbal mental age and to compare imitation delay directly with motor delay. In contrast to non-autistic controls, results of multiple linear regression analyses revealed that in children with autism, imitation delay was predicted by the children's fine motor delay in relation to their nonverbal mental abilities (Vanvuchelen et al., 2010a). To summarise, we did not find full evidence that imitation problems are specific to autism. There is undoubtedly an overlap between mental, motor and imitation abilities. But, while mental and motor impairment may affect imitation performance, they do not seem to explain imitation problems in a sufficient way. 


\section{Are imitation problems universal in autism?}

For the criterion of universality to be met, core deficits are expected to appear during at least one age period in all individuals with the diagnosis, regardless of the severity of autism (Sigman et al., 2004). The meta-analysis of Williams and colleagues (2004) indicated that the size of the autism imitation problems is most apparent in younger age groups (Williams et al., 2004).

The universality criterion has been explored in infants and toddlers with autism. Findings from analyses of prediagnostic home videotapes on symptom onset ([Adrien et al., 1991], [Adrien et al., 1993], [Baranek, 1999], [Osterling and Dawson, 1994], [Osterling et al., 2002] and [Werner et al., 2000]) and follow-up studies ([Charman et al., 1997], [Landa and Garrett-Mayer, 2006], [Lord, 1995], [Sullivan et al., 2007], [Wetherby et al., 2004] and [Zwaigenbaum et al., 2005]) suggest deviant imitative behaviour in infants and toddlers with autism compare to other developmental disabilities. However, results of Vanvuchelen et al.'s study (2010a) in 68 children with autism between 1.9 and 4.5 years of age provide no evidence for a universal imitation delay in relation to the children's nonverbal mental age. Of all children, 72 percent had a bodily imitation and 69 percent a procedural imitation age below their nonverbal mental age. Although the imitation delay was most apparent in the group of children with an IQ above 100, analyses on individual level revealed that this was only true for 87 percent (bodily imitation), respectively 78 percent (procedural imitation) of these children (Vanvuchelen et al., 2010a). In Rogers et al.'s study (2010), a combined group with earl-onset and regressive onset autism as a whole had more failures across the imitation tasks than the comparison group of typically developing and developmentally delayed preschoolers. However, sub analyses revealed that these effects were primarily because of the regression onset group (Rogers et al., 2010). Overall, at group level young children with autism show imitation problems. But, these problems seem not to be universal in autism at early age. There is a considerable variability in imitation performances within young children with autism. We could have expected this natural variation in imitation abilities, because autism is by definition, according to the polythetic-categorical concept of the DSM-IV-TR (American Psychiatric Association, 2000), a heterogeneous, behaviourally defined, neurodevelopmental disorder. Not all listed symptoms are necessary to consider autism in a specific individual. As a result, two different individuals who meet the criteria for autism may have no common symptoms (Krueger \& Bezdjian, 2009). In addition, according to longitudinal and retrospective studies not all children with autism clearly exhibit abnormalities early in life. Up to $50 \%$ may display a typical development or only mild delays until 15-24 months of age, followed by loss of language, communication or social skills ([Landa et al., 2007], [Lord et al., 2004] and [Luyster et al., 2005]).

\section{Are imitation problems persistent in autism?}

Findings of imitation studies in older individuals with autism support the criterion of persistency. Adolescents and adults with autism have subtle imitation problems ([Freitag et al., 2006], [Hobson and Lee, 1999] and [Rogers et al., 1996]). They imitate the essential configuration of an action, but not the proper style, e.g., harsh versus gentle (Hobson \& Lee, 1999). Overall, data on this issue are scarce. Results of the above mentioned studies suggest that imitation problems in individuals with autism are long-lasting and persistent. However, long-term follow-up studies should be conducted to confirm this view.

\section{Do imitation problems precede other autism features?}

According to the criterion precedence, imitation problems should exist prior to the development of the symptoms which they are supposed to explain (Rogers, 1999). Zwaigenbaum and colleagues (2005) provided the first evidence of imitation problems in infants prior to diagnosis of autism, suggesting that imitative deficits may precede development of the full syndrome. They followed infant siblings of children with autism from 6 months to 2 years. At the age of 2 years, 19 out of 65 children presented with symptoms corresponding to autism. Imitation 
items administered at the age of 1 year, along with several other indicators, predicted these 19 children at a $p$ value of 0.003 (Zwaigenbaum et al., 2005).

Longitudinal studies of young children with autism reveal that gestural imitation predicts the development of expressive language (Stone et al., 1997), receptive language (Charman et al., 2003) or both ([Stone and Yoder, 2001] and [Toth et al., 2006]). On the other hand, findings from cross-sectional autism studies have demonstrated that concurrent language is predicted by several nonverbal behaviours, including imitation ([Carpenter et al., 2002], [Luyster et al., 2008] and [Toth et al., 2006]). Thus at the moment findings about the precedence criterion of imitation problems in autism are conflicting and inconclusive.

\section{Are imitation problems broad in preschoolers with autism?}

The requirement that the deficit is broad rather than selective leads to studies that compare imitation performance on bodily and procedural imitation tasks. A core deficit should be present in both forms of imitation. The majority of imitation studies did ask individuals with autism to copy only bodily actions, e.g., facial expressions and gestural postures and movements. Virtually every study, except for two studies which were confounded by ceiling effects ([Charman and Baron-Cohen, 1994] and [Morgan et al., 1989]) has reported bodily imitation problems. This includes studies that tap the full range of age groups and severity levels ([Bartak et al., 1975], [Dewey et al., 2007], [Freitag et al., 2006], [Green et al., 2002], [Ham et al., 2008], [Jones and Prior, 1985], [Mostofsky et al., 2006], [Ohta, 1987], [Rogers et al., 1996], [Smith and Bryson, 1998], [Smith and Bryson, 2007], [Vanvuchelen et al., 2007a] and [Vanvuchelen et al., 2007b]).

In contrast to bodily imitation, research on procedural imitation is less conclusive, varying from robust (Charman et al., 1997), to moderate (Rogers et al., 2010), to no procedural imitation problems in children with autism ([Ingersoll et al., 2003], [McDonough et al., 1997] and [Rogers et al., 2008]). In some studies ([Ingersoll et al., 2003], [McDonough et al., 1997] and [Rogers et al., 2008]) the use of significantly younger typically developing controls may have given the participants with autism an advantage. The younger age of the typically developing children may have resulted in fewer opportunities for imitative learning and less exposure to demanding situations in general. In addition, the fact that some autistic children in the study of Ingersoll received structured imitation training before participation in the study may have improved their imitation ability (Ingersoll et al., 2003).

In fact, only studies that investigate both bodily and procedural imitation in the same individuals with autism compared to appropriate controls have the potential to unravel the question if individuals with autism display broad or selective imitation problems. Research findings of these studies are inconclusive ([Aldridge et al., 2000], [Beadle-Brown and Whiten, 2004], [Carpenter et al., 2002], [DeMeyer et al., 1972], [Hammes and Langdell, 1981], [Libby et al., 1997], [Rogers et al., 2003], [Roeyers et al., 1998], [Stone et al., 1990], [Stone et al., 1997] and [Vanvuchelen et al., 2010a]). Several studies found neither bodily nor procedural imitation problems in children with autism ([Beadle-Brown and Whiten, 2004] and [Carpenter et al., 2002]) or no procedural imitation problems, because their results were confounded by ceiling effects (Hammes \& Langdell, 1981). Most studies found that mentally impaired children with autism performed significantly poorer during bodily and procedural imitation than developmentally delayed controls ([DeMeyer et al., 1972], [Roeyers et al., 1998], [Stone et al., 1990] and [Stone et al., 1997]) and younger typically developing children ([Stone et al., 1990] and [Stone et al., 1997]). Group differences on bodily imitation were of a greater magnitude than these on procedural imitation ([DeMeyer et al., 1972], [Roeyers et al., 1998] and [Stone et al., 1997]). In contrast, the research group of Aldridge (2000) concluded that mentally impaired children with autism were poorer on bodily imitation, but better on procedural imitation than mental age-matched typically developing infants (Aldridge et al., 2000). Rogers and colleagues (2003) found another pattern in children with autism compared to chronological and mental age-matched nonautistic controls. The children with autism showed impairments in facial and procedural imitation, but not in gestural imitation (Rogers et al., 2003). Within group analyses in Vanvuchelen et al.'s study (2010a) revealed 
clear bodily and procedural imitation delay in the children with autism. There was no evidence for a difference between bodily and procedural imitation delay (Vanvuchelen et al., 2010a). Finally, the research group of Libby (1997) investigated imitation performances on pretended play acts with and without objects in children with autism, younger children with Down syndrome and younger typically developing children. While the autistic children were better than the other two groups on single-scheme procedural and bodily imitation tasks, they demonstrated specific difficulties on the multischeme tasks (Libby et al., 1997). Overall, at the moment findings about the broadness criterion of imitation problems in autism are inconclusive.

\section{Conclusion}

A critical conclusion of the present literature review is that imitation problems are a less robust core deficit in autism than previously suggested ([Rogers and Pennington, 1991], [Rogers, 1999], [Smith and Bryson, 1994] and [Williams et al., 2004]). Findings of this review suggest that there is only partial evidence that imitation problems are unique, specific and broad to autism. The clear overlap between mental, motor and imitation abilities is well documented. Mental and motor impairment may affect imitation performance, but they do not appear to explain autism imitation problems in a sufficient way. Although data about the persistency criterion are scarce, autism imitation problems may be long-lasting and persistent. Further work is required to clarify this issue. Furthermore, there is some evidence that early imitation problems with several other indicators precede and predict the diagnosis of autism. Less evidence is found for the idea that imitation problems are universal in autism at early age.

Three recommendations for clinical practice can be made. First, the imitation abilities of all persons who are suspected of having imitation problems should be assessed. Imitation assessment may offer meaningful information about the future learning capacity of these persons and accordingly about their possible response to treatment and developmental outcome ([Rogers et al., 2006] and [Sallows and Graupner, 2005]). This is in contrast with most developmental tests, which investigate what children have achieved so far. Second, imitation abilities should be assessed in conjunction with the assessment of mental and motor competences. Third, imitation problems should be defined in accordance with the persons' mental age. This kind of dimensional data may advance the understanding of the phenotypic heterogeneity in autism. In addition, a better sample specification through ratings of imitation problems, may allow a more personalised clinical diagnosis and treatment of persons with autism. As no clearly defined behavioural or developmental intervention improved all symptoms for all individuals with autism, the clinical management should be guided by individual needs and availability of resources (Ospina et al., 2008). The possibility to distinguish a person with imitation problems from one without imitation problems, may allow us to refer the appropriate person for appropriate intervention programs, such as imitation training programs.

\section{Acknowledgments}

This study was part of the doctoral dissertation of M. Vanvuchelen and supported by the Foundation MargueriteMarie Delacroix (Tienen, Belgium). 


\section{References}

Adrien, J. L., Faure, M., Perrot, A., Hameury, L., Garreau, B., Barthelemy, C., et al. (1991). Autism and family home movies: Preliminary findings. Journal of Autism and Developmental Disorders, 21, 43-49.

Adrien, J. L., Lenoir, P., Martineau, J., Perrot, A., Hameury, L., Larmande, C., et al. (1993). Blind ratings of early symptoms of autism based upon family home movies. Journal of the American Academy of Child and Adolescent Psychiatry, 32, 617-626.

American Psychiatric Association. (2000). Diagnostic and statistical manual of mental disorders DSM-IV-TR (Text Revison). Washington DC: American Psychiatric Association.

Aldridge, M. A., Stone, K. R., Sweeney, M. H., \& Bower, T. G. R. (2000). Preverbal children with autism understand the intentions of others. Developmental Science, 3, 294-301.

Baranek, G. T. (1999). Autism during infancy: A retrospective video analysis of sensory-motor and social behaviors at 9-12 months of age. Journal of Autism and Developmental Disorders, 29, 213-224.

Bartak, L., Rutter, M., \& Cox, A. (1975). A comparative study of infantile autism and specific development receptive language disorder. I. The children. British Journal of Psychiatry, 126, 127-145.

Beadle-Brown, J., \& Whiten, A. (2004). Elicited imitation in children and adults with autism: Is there a deficit? Journal of Intellectual \& Developmental Disability, 29, 147-163.

Bird, G., Leighton, J., Press, C., \& Heyes, C. (2007). Intact automatic imitation of human and robot actions in autism spectrum disorders. Proceedings of the Royal Society B, 274, 3027-3031.

Carpenter, M., Pennington, B. F., \& Rogers, S. J. (2002). Interrelations among social-cognitive skills in young children with autism. Journal of Autism and Developmental Disorders, 32, 91-106.

Charman, T., \& Baron-Cohen, S. (1994). Another look at imitation in autism. Development and Psychopathology, $6,403-413$.

Charman, T., Swettenham, J., Baron-Cohen, S., Cox, A., Baird, G., \& Drew, A. (1997). Infants with autism: An investigation of empathy, pretend play, joint attention, and imitation. Developmental Psychology, 33, 781-789.

Charman, T., Baron-Cohen, S., Swettenham, J., Baird, G., Drew, A., \& Cox, A. (2003). Predicting language outcome in infants with autism and pervasive developmental disorder. International Journal of Language and Communication Disorders, 38, 265-285.

DeMeyer, M. K., Alpern, G. D., Barton, S., DeMyer, W. E., Churchill, D. W., Hingtgen, J. N., et al. (1972). Imitation in autistic, early schizophrenic, and non-psychotic subnormal children. Journal of Autism and Childhood Schizophrenia, 2, 264-287.

Dewey, D., Cantell, M., \& Crawford, S. G. (2007). Motor and gestural performance in children with autism spectrum disorders, developmental coordination disorder, and/or attention deficit hyperactivity disorder. Journal of the International Neuropsychological Society, 13, 246-256. 
Dziuk, M. A., Gidley Larson, J. C., Apostu, A., Mahone, E. M., Denckla, M. B., \& Mostofsky, S. H. (2007). Dyspraxia in autism: Association with motor, social, and communicative deficits. Developmental Medicine and Child Neurology, 49, 734-739.

Fournier, K.A., Hass, C.J., Naik, S.K., Lodha, N., Cauraugh, J.H. (2010). Motor coordination in autism spectrum disorders: A synthesis and meta-analysis. Journal of Autism and Developmental Disorders, in press, doi:10.1007/s10803-010-0981-3.

Freitag, C. M., Kleser, C., \& Von Gontard, A. (2006). Imitation and language abilities in adolescents with Autism Spectrum Disorder without language delay. European Child and Adolescent Psychiatry, 15, 282-291.

Green, D., Baird, G., Barnett, A. L., Henderson, L., Huber, J., \& Henderson, S. E. (2002). The severity and nature of motor impairment in Asperger's syndrome: A comparison with specific developmental disorder of motor function. Journal of Child Psychology and Psychiatry, 43, 655-668.

Ham, S. H. C. M. , Rajendran, G., \& Carletta, J. (2008). Brief report: Imitation of meaningless gestures in individuals with Asperger syndrome and high-functioning autism. Journal of Autism and Developmental Disorders, 38, 569-573.

Hammes, J. G., \& Langdell, T. (1981). Precursors of symbol formation and childhood autism. Journal of Autism and Developmental Disorders, 11, 331-346.

Hobson, R. P., \& Lee, A. (1999). Imitation and identification in autism. Journal of Child Psychology and Psychiatry, 40, 649-659.

Ingersoll, B., Schreibman, L., \& Tran, Q. H. (2003). Effect of sensory feedback on immediate object imitation in children with autism. Journal of Autism and Developmental Disorders, 33, 673-683.

Ingersoll, B. (2008). The effect of context on imitation skills in children with autism. Research in Autism Spectrum Disorders, 2, 332-340.

Jaeschke, R., Guyatt, G., \& Sackett, D. L. (1994). Users' guides to the medical literature. III. How to use an article about a diagnostic test. A. Are the results of the study valid? Evidence-based medicine working group. The Journal of the American Medical Association, 271, 389-391.

Jones, V., \& Prior, M. (1985). Motor imitation abilities and neurological signs in autistic children. Journal of Autism and Developmental Disorders, 15, 37-46.

Knott, F., Lewis, C., \& Williams, T. (2007). Sibling interaction of children with autism: Development over 12 months. Journal of Autism and Developmental Disorders, 37, 1987-1995.

Krueger, R. F., \& Bezdjian, S. (2009). Enhancing research and treatment of mental disorders with dimensional concepts: Toward DSM-V and ICD-11. World Psychiatry, 8(1), 3-6.

Landa, R., \& Garrett-Mayer, E. (2006). Development in infants with autism spectrum disorders: A prospective study. Journal of Child Psychology and Psychiatry, 47, 629-638.

Landa, R. J., Holman, K. C., \& Garrett-Mayer, E. (2007). Social and communication development in toddlers with early and later diagnosis of autism spectrum disorders. Archives of General Psychiatry, 64, 853-864. 
Levy, S. E., Mandell, D. S., \& Schultz, R. T. (2009). Autism. Lancet, 374, 1627-1638.

Libby, S., Powell, S., Messer, D., \& Jordan, R. (1997). Imitation of pretend play acts by children with autism and Down syndrome. Journal of Autism and Developmental Disorders, 27, 365-383.

Lord, C. (1995). Follow-up of two-year-olds referred for possible autism. Journal of Child Psychology and Psychiatry, 36, 1365-1382.

Lord, C., Shulman, C., \& DiLavore, P. (2004). Regression and word loss in autistic spectrum disorders. Journal of Child Psychology and Psychiatry, 45, 936-955.

Luyster, R., Richler, J., Risi, S., Hsu, W. L., Dawson, G., Bernier, R., et al. (2005). Early regression in social communication in autism spectrum disorders: A CPEA Study. Developmental Neuropsychology, 27, 311-336.

Luyster, R. J., Kadlec, M. B., Carter, A., \& Tager-Flusberg, H. (2008). Language assessment and development in toddlers with autism spectrum disorders. Journal of Autism and Developmental Disorders, 38, 1426-1438.

McDonough, L., Stahmer, A., Schreibman, L., \& Thompson, S. J. (1997). Deficits, delays, and distractions: An evaluation of symbolic play and memory in children with autism. Development and Psychopathology, 9, 17-41.

McDuffie, A., Turner, L., Stone, W., Yoder, P., Wolery, M., \& Ulman, T. (2007). Developmental correlates of different types of motor imitation in young children with autism spectrum disorders. Journal of Autism and Developmental Disorders, 37, 401-412.

Mclntosh, D. N., Reichmann-Decker, A., Winkielman, P., \& Wilbarger, J. L. (2006). When the social mirror breaks: Deficits in automatic, but not voluntary, mimicry of emotional facial expressions in autism. Developmental Science, 9, 295-302.

Morgan, S. B., Cutrer, P. S., Coplin, J. W., \& Rodrigue, J. R. (1989). Do autistic children differ from retarded and normal children in Piagetian sensorimotor functioning? Journal of Child Psychology and Psychiatry, 30, 857-864.

Mostofsky, S. H., Dubey, P., Jerath, V. K., Jansiewicz, E. M., Goldberg, M. C., \& Denckla, M. B. (2006). Developmental dyspraxia is not limited to imitation in children with autism spectrum disorders. Journal of the International Neuropsychological Society, 12, 314-326.

Ohta, M. (1987). Cognitive disorders of infantile autism: A study employing the WISC, spatial relationship conceptualization, and gesture imitations. Journal of Autism and Developmental Disorders, 17, 45-62.

Ospina, M. B., Krebs, S. J., Clark, B., Karkhaneh, M., Hartling, L., Tjosvold, L., et al. (2008). Behavioural and developmental interventions for autism spectrum disorder: A clinical systematic review. PLoS One, 3, e3755.

Osterling, J., \& Dawson, G. (1994). Early recognition of children with autism: A study of first birthday home videotapes. Journal of Autism and Developmental Disorders, 24, 247-257.

Osterling, J. A., Dawson, G., \& Munson, J. A. (2002). Early recognition of 1-year-old infants with autism spectrum disorder versus mental retardation. Development and Psychopathology, 14, 239-251.

Perra, O., Williams, J. H. G., Whiten, A., Fraser, L., Benzie, H., \& Perrett, D. I. (2008). Imitation and 'theory of mind' competencies in discrimination of autism from other neurodevelopmental disorders. Research in Autism Spectrum Disorders, 2, 456-468. 
Prinz, W. (2002). Experimental approaches to imitation. In A. N. Meltzoff \& W. Prinz (Eds.), The imitative mind: Development, evolution, and brain bases (pp. 143-162). Cambridge: Cambridge University Press.

Roeyers, H., Van Oost, P., \& Bothuyne, S. (1998). Immediate imitation and joint attention in young children with autism. Development and Psychopathology, 10, 441-450.

Rogers, S. J., \& Pennington, B. F. (1991). A theoretical approach to the deficits in infantile autism. Development and Psychopathology, 3, 137-162.

Rogers, S. J., Bennetto, L., McEvoy, R., \& Pennington, B. F. (1996). Imitation and pantomime in high-functioning adolescents with autism spectrum disorders. Child Development, 67, 2060-2073.

Rogers, S. J. (1999). An examination of the imitation deficit in autism. In J. Nadel \& G. Butterworth (Eds.), Imitation in Infancy (pp. 254-283). Cambridge: Cambridge University Press.

Rogers, S. J., Hepburn, S. L., Stackhouse, T., \& Wehner, E. (2003). Imitation performance in toddlers with autism and those with other developmental disorders. Journal of Child Psychology and Psychiatry, 44, 763-781.

Rogers, S. J., Hayden, D., Hepburn, S., Charlifue-Smith, R., Hall, T., \& Hayes, A. (2006). Teaching young nonverbal children with autism useful speech: A pilot study of the Denver Model and PROMPT interventions. Journal of Autism and Developmental Disorders, 36, 1007-1024.

Rogers, S. J., Young, G. S., Cook, I., Giolzetti, A., \& Ozonoff, S. (2008). Deferred and immediate imitation in regressive and early onset autism. Journal of Child Psychology and Psychiatry, 49, 449-457.

Rogers, S. J., Young, G. S., Cook, I., Giolzetti, A., \& Ozonoff, S. (2010). Imitating actions on objects in early-onset and regressive autism: Effects and implications of task characteristics on performance. Development and Psychopathology, 22(1), 71-85.

Sallows, G. O., \& Graupner, T. D. (2005). Intensive behavioral treatment for children with autism: Four-year outcome and predictors. American Journal of Mental Retardation, 110, 417-438.

Sevlever, M., \& Gillis, J. M. (2010). An examination of the state of imitation research in children with autism: Issues of definition and methodology. Research in Developmental Disabilities, 31, 976-984.

Sigman, M., Dijamco, A., Gratier, M., \& Rozga, A. (2004). Early detection of core deficits in autism. Mental retardation and developmental disabilities research reviews, 10, 221-233.

Smith, I. M., \& Bryson, S. E. (1994). Imitation and action in autism: A critical review. Psychological Bulletin, 116, 259-273.

Smith, I. M., \& Bryson, S. E. (1998). Gesture imitation in autism I: Nonsymbolic postures and sequences. Cognitive Neuropsychology, 15, 747-770.

Smith, I. M., \& Bryson, S. E. (2007). Gesture imitation in autism. II. Symbolic gestures and pantomimed object use. Cognitive Neuropsychology, 24, 679-700.

Stone, W. L., Lemanek, K. L., Fishel, P. T., Fernandez, M. C., \& Altemeier, W. A. (1990). Play and imitation skills in the diagnosis of autism in young children. Pediatrics, 86, 267-272. 
Stone, W. L., Ousley, O. Y., \& Littleford, C. D. (1997). Motor imitation in young children with autism: What's the object? Journal of Abnormal Child Psychology, 25, 475-485.

Stone, W. L., \& Yoder, P. J. (2001). Predicting spoken language level in children with autism spectrum disorders. Autism, 5, 341-361.

Sullivan, M., Finelli, J., Marvin, A., Garrett-Mayer, E., Bauman, M., \& Landa, R. (2007). Response to joint attention in toddlers at risk for autism spectrum disorder: A prospective study. Journal of Autism and Developmental Disorders, 37, 37-48.

Thurm, A., Lord, C., Lee, L. C., \& Newschaffer, C. (2007). Predictors of language acquisition in preschool children with autism spectrum disorders. Journal of Autism and Developmental Disorders, 37, 1721-1734.

Toth, K., Munson, J., Meltzoff, A. N., \& Dawson, G. (2006). Early predictors of communication development in young children with autism spectrum disorder: Joint attention, imitation, and toy play. Journal of Autism and Developmental Disorders, 36, 993-1005.

Tomasello, M., Carpenter, M., Call, J., Behne, T., \& Moll, H. (2005). Understanding and sharing intentions: The origins of cultural cognition. Behavioral and Brain Sciences, 28, 675-691.

Vanvuchelen, M., Roeyers, H., \& De Weerdt, W. (2007a). Nature of motor imitation problems in school-aged boys with autism: A motor or a cognitive problem? Autism, 11, 225-240.

Vanvuchelen, M., Roeyers, H., \& De Weerdt, W. (2007b). Nature of motor imitation problems in school-aged males with autism: How congruent are the error types? Developmental Medicine and Child Neurology, 49, 6-12.

Vanvuchelen, M., Roeyers, H., De Weerdt, W. (2010a). Imitation assessment and its utility to the diagnosis of autism: Evidence from consecutively clinical preschool referrals for suspected autism, Journal of Autism and Developmental Disorders, in press, doi:10.1007/s10803-010-1074-z.

Vanvuchelen, M., Roeyers, H., \& De Weerdt, W. (2010b). Development and initial validation of the Preschool Imitation and Praxis Scale (PIPS). Research in Autism Spectrum Disorders, in press, doi:10.1016/j.rasd.2010.06.010.

Warreyn, P., Roeyers, H., \& De Groote, I. (2005). Early social communicative behaviours of preschoolers with autism spectrum disorder during interaction with their mothers. Autism, 9, 342-361.

Werner, E., Dawson, G., Osterling, J., \& Dinno, N. (2000). Brief report: Recognition of autism spectrum disorder before one year of age: A retrospective study based on home videotapes. Journal of Autism and Developmental Disorders, 30, 157-162.

Wetherby, A. M., Woods, J., Allen, L., Cleary, J., Dickinson, H., \& Lord, C. (2004). Early indicators of autism spectrum disorders in the second year of life. Journal of Autism and Developmental Disorders, 34, 473-493.

Whiten, A., \& Brown, J. D. (1999). Imitation and the reading of other minds: Perspectives from the study of autism, normal children and non-human primates. In S.

Braten (Ed.), Intersubjective communication and emotion in early ontogeny (pp. 260-280). Cambridge: Cambridge University Press. 
Williams, J. H., Whiten, A., \& Singh, T. (2004). A systematic review of action imitation in autistic spectrum disorder. Journal of Autism and Developmental Disorders, 34, 285-299.

Zachor, D. A., llanit, T., \& Itzchak, E. B. (2010). Autism severity and motor abilities correlates of imitation situations in children with autism spectrum disorders. Research in Autism Spectrum Disorders, 4, 438-443.

Zwaigenbaum, L., Bryson, S., Rogers, T., Roberts, W., Brian, J., \& Szatmari, P. (2005). Behavioral manifestations of autism in the first year of life. International journal of developmental neuroscience, 23, 143-152. 